
\title{
ANÁLISIS DEL PERFIL Y SITUACIÓN CONTRACTUAL DE LOS AGENTES DE EMPLEO Y DESARROLLO LOCAL EN EL CONTEXTO DE LA GESTIÓN TURÍSTICA. AYUNTAMIENTOS DE LA PROVINCIA DE A CORUÑA
}

\author{
PÉREZ GARCÍA Antonia \\ EUT-UDC ${ }^{1}$ \\ antonia.perezg@udc.es
}

Material original autorizado para su primera publicación en la revista académica REDMARKA. Revista Digital de Marketing Aplicado. https://doi.org/10.17979/redma.2016.01.017.4862

Recibido: 19 Septiembre 2016

Aceptado: 20 Diciembre 2016

\section{Resumen}

Los Agentes de Empleo y Desarrollo Local constituyen un sujeto clave en la gestión y dinamización de las políticas activas de desarrollo en el territorio local: impulsando y ejecutando proyectos y acciones que tienen como objetivos crear empleo, fomentar iniciativas empresariales y estimular la promoción económica municipal. Por lo tanto, la AEDL se configuró como una de principales plataformas de apoyo para el impulso de la economía local del municipio, entre las que destaca el sector turístico, por lo que estaríamos hablando de aquellas actividades o iniciativas locales relacionadas con este sector productivo.

Uno de los objetivos, el cual se presenta en este artículo, es analizar el perfil y la situación contractual de los AEDLs, en dicho contexto de gestión turística, centrado en los ayuntamientos de la provincia de A Coruña; para el cual se ha

1 Profesora titular de la Escuela Universitaria de Turismo-CENP, adscrita a la Universidade da Coruña, de las asignaturas de Sociología del Turismo y Métodos y Técnicas de Investigación Social. Doctoranda en el programa Análisis Económico y Estrategia Empresarial en la Facultad de Economía y Empresa-UDC. Autora de la tesis doctoral, en proceso de depósito, "Modelos y técnicas de Relaciones Públicas aplicables a las Agencias de Empleo y Desarrollo Local en el marco de la gestión y promoción turística en la provincia de A Coruña".

REDMARKA UIMA-Universidad de A Coruña - CIECID

Año IX, Número 17, (2016), v I pp. 5-33

http://www.redmarka.net/ ISSN 1852-2300 
aplicado el método exploratorio-descriptivo basado en una investigación metodológica mixta de tipo cualitativo y cuantitativo.

Los resultados extraídos han permitido, con posterioridad, continuar investigando sobre las estrategias informativo-comunicativas de relaciones públicas, de forma que han supuesto una de las bases principales para comprender el contexto en el que trabajan los AEDLs.

Palabras claves: Turismo, Desarrollo Local, Agente de Empleo y Desarrollo Local (AEDL) y A Coruña.

\section{Abstract}

Employment and Local Development Agents play a key role in the management and dynamization of active development policies in the local territory: promoting and executing projects and actions designed to generate employment, encouraging business initiatives and stimulating municipal economic promotion. ELDAs were designed as one of the main support platforms for boosting the local economy of a municipality, with particular emphasis on the tourism sector; in other words, local activities or initiatives related to this industry.

One of the objectives which is presented in this article is to analyze the profile and the contractual situation of ELDAs in the context of tourist management, centered in the municipalities of the province of A Coruña. An exploratorydescriptive method has been applied based on a mixed methodological research of a qualitative and quantitative nature.

The results obtained have allowed for ongoing research into informativecommunicative public relations strategies, providing a key insight into the context of ELDAs.

Key words: Tourism, Local Development, Employment and Local Development Agent (ELDA) and A Coruña.

\section{Introducción}


En relación al desarrollo local Galicia emerge, desde los años ochenta, como un actor con grandes potencialidades endógena: posee una importante línea de costa, unos recursos naturales protegidos, atesora un importante legado histórico-artístico, aguas termales y un gran valor cultural en su conjunto (Díaz Fernández, Hernández Borge y Patiño Romarís, 2000); inmersos en este contexto, las AEDLs han ejercido un papel relevante a nivel turístico en busca de una mejora del desarrollo económico y social como bien público, a pesar de las dificultades financieras y económicas padecidas por la situación de crisis que arrastramos desde hace un tiempo. Lo cierto es que, en la última década, los cambios sociales y económicos han aportado la implantación y gestión de una concepción diferente sobre la función del ocio y del turismo, partiendo de este escenario, uno de los objetivos de este estudio ha sido explorar y describir características y situación actual que han estimulado una forma de gestionar el turismo por parte de las AEDLs, en la provincia de A Coruña. Del análisis de este primer objetivo se han obtenido resultados y conclusiones interesantes que evidencian una particular situación coyuntural, desde hace algún tiempo, con respecto a los AEDLs en la provincia de A Coruña, la cual creemos es extensiva a toda Galicia, ya que parece no haber una definición clara sobre la política de desarrollo local en materia de turismo.

\section{Revisión de la literatura}

En ésta se presenta un resumen del marco teórico, aquella que permite comprender las funciones, características, normativa y situación actual de las AEDLs en general, y en particular en Galicia. También serán revisados los aspectos teóricos más relevantes relativos al desarrollo local y el turismo.

\subsection{Antecedentes, origen y normativa sobre las agencias y los Agentes de Empleo y Desarrollo Local}

Los Agentes de Empleo y Desarrollo Local se articulan como un sujeto clave en la dinamización de las políticas activas de empleo en el territorio local, impulsando y gestionando proyectos y acciones que tienen como objetivo la 
creación de empleo y el fomento de la actividad empresarial. Los ayuntamientos se han visto en la necesidad de implantar un profesional que fuese capaz de programar y gestionar este objetivo, y es aquí donde aparece la figura del Agente de Empleo y Desarrollo Local, gestor que sea capaz de aprovechar, tanto los recursos endógenos como las ayudas procedentes de programas europeos. La AEDL se constituye como la principal plataforma de apoyo para el impulso de la economía del municipio, a la vez que promotor, junto con otros servicios municipales, de la dinamización social de la comunidad local.

En un documento publicado por Feprodel (Federación Nacional de Profesionales de Desarrollo Local) en marzo de 2011 sobre la "Situación Laboral y Profesional de los AEDL", aparece un resumen sobre las primeras iniciativas de desarrollo local y el origen de las ADLs, en este caso es interesante extraer y comentar parte de este documento con el objeto de realizar una aproximación a la realidad profesional actual de los mismos.

Será en el Reino Unido en 1973 donde surjan las primeras Agencias de Desarrollo Local en respuesta a la crisis energética mundial que generó un importante nivel de desempleo, éstas tenían el objetivo de diseñar nuevos modelos de crecimiento económico. Más tarde, en 1982, la OCDE pone en marcha un Programa para el Desarrollo Económico y el Empleo Local que tendrá por objeto la identificación, el análisis y la difusión de ideas reformadoras sobre desarrollo económico local y de creación de empleo. En este programa se ponía el énfasis en la dimensión local y llegaron a participar hasta trece países europeos, entre los que se encontraba España. Finalmente, cuando España se incorpora a la CEE en 1986, se publica la Orden del Ministerio de Trabajo de 21 de febrero de 1986, en vigor hasta 1994, en la que se establecen diversos programas de apoyo a la creación de empleo. El Programa II hace referencia a la promoción de las "iniciativas locales para la creación de empleo", teniendo como meta impulsar y financiar propuestas que generen empleo estable mediante la creación de pequeñas o medianas empresas que utilicen recursos ociosos en la localidad o comarca. 
Se puede afirmar pues que el profesional de Agente de Empleo y Desarrollo Local, tal y como se entiende actualmente, fue promovido en sus orígenes por la Unión Europea y orientado a través del Fondo Social Europeo, con el objetivo de promover el desarrollo local dentro de la agenda municipal, además de incluir a estos profesionales como una figura estable dentro de la estructura de la institución, a medida que los ayuntamientos iban contemplando el desarrollo local como una función necesaria.

En el marco de los convenios firmados entre el Ministerio de Trabajo y las Comunidades Autónomas o la Federación Española de Municipios y Provincias (FEMP), se podían conceder subvenciones para la contratación de Agentes de Desarrollo o profesionales con similar función, pero esta orden no establece titulación universitaria para la realización de dichas funciones y se llegaron a contratar hasta un total de trescientos profesionales en toda España.

Posteriormente, la Orden del Ministerio de Trabajo de 12 de abril de 1994 que estará en vigor hasta 1999, regulará las iniciativas locales de empleo y de desarrollo local, y se establecen las bases para la regulación de la concesión de subvenciones para dichas iniciativas y para la contratación de los AEDL, ya que esta orden contempla, por primera vez, la designación de Agente de Empleo y Desarrollo Local (AEDL.

Cinco años más tarde, en concreto en 1999, acontece la descentralización de los Servicios Públicos de Empleo y se publica la Orden del Ministerio de Trabajo de 15 de julio de 1999, en vigor hasta el año 2008, en la cual se establecen las bases de concesión de subvenciones públicas para el fomento del desarrollo local e impulso de los proyectos y empresas calificados como I+E, quedando desvinculada la función de las iniciativas locales de empleo pero con vinculación a la creación de actividad empresarial. Esta nueva orden introducirá novedades en las funciones, anteriormente establecidas, como serán:

- La colaboración en la promoción e implantación de las políticas activas de empleo relacionadas con la creación de actividad empresarial. 
- Una vez constituidas las empresas, se ofrece apoyo y acompañamiento técnico durante las primeras fases de funcionamiento -consultoría técnica en gestión empresarial y asistencia técnica en los procesos formativos-.

La última Orden en vigor hasta la actualidad fue publicada el 6 de febrero de 2008 (Orden TAS/360/2008), orden que modifica la de 1999, en la que se establecen las bases de concesión de subvenciones públicas para la promoción del desarrollo local y permite que los contratos de los AEDLs se puedan renovar todos los años, suprimiendo la restricción de cuatro años que establecía la anterior orden ministerial.

Será a partir de este momento, cuando cada Servicio Regional de Empleo, al publicar su convocatoria anual de subvención, incluirá funciones específicas acordes a las necesidades de su territorio y baremos distintos para puntuar los proyectos presentados, lo que provocará diferencias importantes sobre el porcentaje total a subvencionar, esto afectará, específicamente, sobre las prórrogas de los contratos de los AEDLs ya que estas contrataciones y sus prorrogas estarán supeditadas a la disponibilidad de la financiación que viene dada por el Servicio Regional de Empleo de cada Comunidad Autónoma.

También hay que tener en cuenta que en la evolución y en el avance de esta regulación se ha ido incorporando la dimensión de lo "local", que destacará la Unión Europea en la Comunicación de la Comisión: "Actuación local en favor del empleo: una dimensión local para la Estrategia Europea de Empleo" (COM, 2000, p. 196), en la cual se establecen los siguientes factores a tener en cuenta:

-Dimensión local de la política de empleo, ajustadas a las necesidades del territorio de forma que incentiven y generen la creación de empleo (Ley 56/2003 de 16 de diciembre, R.D. Ley 3/2011 de 18 de febrero).

-Enfoque ascendente.

-Financiación adaptada a las necesidades locales.

-Políticas económicas, estructurales y sociales que se refuercen mutuamente. 


\subsection{Función de los Agentes de Desarrollo Local}

EI AEDL desarrolla y ejerce fundamentalmente una serie de funciones como son (Redondo Rodríguez de la Vera y colaboradores, 2000, pp. 27-28):

- Animador social con el objetivo de obtener participación e iniciativa ciudadana.

- Informador y facilitador de apoyo técnico para que los proyectos y programas de actuación sean exitosos.

- Mediador entre los diferentes actores sociales locales que intervienen en los proyectos e iniciativas.

- Conocedor del medio y de la realidad social a través del análisis y la experiencia propia.

En el Manual del Agente de Desarrollo Local de Alburqueque (1999, p. 29) encontramos una serie de herramientas de orientación dirigidas a los AEDL. De manera muy resumida viene a resaltar algunos aspectos que debería tener en cuenta este profesional:

- El AEDL es un consultor local ya que ha de desarrollar iniciativas de carácter público.

- Necesita tener conocimientos básicos de la realidad social en la que debe actuar.

- Es tan importante lo que hace como el cómo lo hace por lo que ha de ser una persona dinámica, sociable, interactiva y con gran capacidad de adaptación.

- Debe tener una visión y pensamiento estratégico para abordar la planificación de las iniciativas.

Vicente Martín en Rodríguez Gutiérrez (2001, pp. 473-482) expone el perfil que debería tener el agente de desarrollo local porque el éxito de la puesta en marcha de las iniciativas locales depende de sus conocimientos en diversas áreas ya que es "una tarea compleja desde un lugar pequeño" ( $p$. 485). A continuación resumimos los rasgos y serían los siguientes: 


\section{Tabla 1}

El perfil de Agente de Desarrollo Local

\begin{tabular}{|c|c|}
\hline ÁREA & CONOCIMIENTOS \\
\hline $\begin{array}{l}\text { Análisis } \\
\text { territorial }\end{array}$ & $\begin{array}{l}\text { - Entorno territorial de intervención. } \\
\text { - } \quad \text { Marco natural y geográfico. } \\
\text { - Marco urbanístico o de ordenación del territorio. } \\
\text { - Marco micro y macro economía: Mercado de } \\
\text { trabajo, consumo, corrientes migratorias, etc. }\end{array}$ \\
\hline $\begin{array}{l}\text { Promoción } \mathrm{de} \\
\text { proyectos }\end{array}$ & $\begin{array}{l}\text { - Metodología necesaria para programar y aplicar } \\
\text { el proyecto. } \\
\text { - Comprensión y capacidad de explicación a la } \\
\text { población local. }\end{array}$ \\
\hline $\begin{array}{l}\text { Animación } \quad \text { y } \\
\text { relaciones } \\
\text { humanas }\end{array}$ & $\begin{array}{l}\text { - } \quad \text { Comprensión de los fenómenos sociales. } \\
\text { - } \quad \text { Liderazgo. } \\
\text { - } \quad \text { Capacidad de influencia en caso necesario. } \\
\text { - }\end{array}$ \\
\hline Comunicación & $\begin{array}{l}\text { - Estrategias modernas de comunicación. } \\
\text { - Instrumentos y técnicas de comunicación como } \\
\text { apoyo a las acciones. } \\
\text { - Medios de Comunicación y soportes } \\
\text { comunicacionales. }\end{array}$ \\
\hline Gestión & $\begin{array}{l}\text { - Técnicas modernas contables. } \\
\text { - Legislación sobre derecho laboral, administrativo } \\
\text { y comercial. } \\
\text { - Gestión de administración. } \\
\text { - Manipulación del lenguaje y técnicas propias de } \\
\text { las administraciones. }\end{array}$ \\
\hline
\end{tabular}

Elaboración propia. Fuente: En Rodríguez Gutiérrez (2001) Vicente Martín, I. Capítulo "El agente de desarrollo local" (pp. 473-482)

En cuanto a las funciones que ha de desarrollar el AEDL, Vicente Martín enfatiza cuatro concretamente:

- Realización de estudios e investigaciones precedentes sobre las estructuras administrativas que intervienen en el territorio, posibles potencialidades locales endógenas y estudio territorial del entorno.

- Localizar recursos turísticos, y de otra naturaleza, endógenos inéditos, creación y explotación de nuevos productos, así como buscar nuevas formas de comercialización e información. Aquí tienen las relaciones públicas un papel esencial que, aunque es una forma REDMARKA UIMA-Universidad de A Coruña-CIECID

Año IX, Número 17, (2016), v I pp. 5-33

http://www.redmarka.net/ ISSN 1852-2300 
tradicional de información, es una estrategia desconocida por muchos de los AEDL, no por la utilización de las diferentes técnicas, sino más bien por el desconocimiento sobre los posibles beneficios que puede reportar al resultado final.

- Captar posibles recursos exógenos en un intento de reforzar los medios y la logística necesaria para implementar los proyectos y acciones de desarrollo local.

- Programar teniendo en cuenta la participación y el debate desde la comunidad local, además de trabajar con una visión estratégica de cooperación permanente entre los diferentes actores sociales implicados.

Vemos que la idea de la cooperación y la participación de la comunidad local, así como de otros agentes intervinientes, son reiterativas en los discursos de los autores vistos para la revisión del marco teórico, por lo que claramente afirmamos ser elementos claves, actualmente, en las políticas de desarrollo estratégicas.

Sobre las dificultades a las que el AEDL tiene que enfrentarse, Vázquez Barquero, A. (2000, p. 29-32) insiste en que hay que tener en cuenta una serie de carencias importantes que le limitan su labor como son:

- "Inexistencia de un marco legal adecuado". Vázquez Barquero afirma que no siempre se ha aceptado el desarrollo local como una política de Estado, su origen ha sido más una respuesta espontánea de los propios ayuntamientos ante las carencias detectadas.

- "Reducida autonomía para la acción local", quedando reducida a las localidades más dinámicas y con un papel más emprendedor. La financiación, en la mayor parte de los casos es insuficiente e inadecuada, lo que impide poner en marcha acciones para el desarrollo local y promover y potenciar recursos endógenos.

- "Insuficiencia de recursos humanos para la gestión local". Vázquez Barquero la destaca como una de las limitaciones más importantes. Carecer de 
suficiente personal en la AEDL y también de la cualificación mínima necesaria para gestionar adecuadamente la AEDL.

- "Falta de masas críticas en las unidades territoriales". Las unidades territoriales han de disponer de una dimensión administrativa suficiente, pero no siempre es así por lo que se tiene que recurrir a sinergias como la cooperación entre municipios, colaboración con asociaciones, etc., en busca de una mejor eficiencia.

- "Insuficiente coordinación entre agentes". El éxito de las políticas de desarrollo local depende, en gran medida, de la colaboración y muchas veces se desconoce la importancia de la cooperación a distintos niveles (local, regional, nacional e incluso internacional).

- "El reparto del poder entre los niveles administrativos". La lenta descentralización ha provocado retrasos en la adquisición, por parte de los gobiernos locales y regionales, de competencias y responsabilidades.

\subsection{Historia y el papel de los Agentes de Desarrollo Local en Galicia hasta la actualidad}

En la web de Afiprodel (Asociación Finisterrae de Profesionales de Desarrollo Local de Galicia) se halla información sobre la historia de los AEDLs en Galicia, en la cual se indica que las primeras Agencias de Desarrollo Local ( $A D L)$ en los ayuntamientos de Galicia aparecen en 1989, dentro de una convocatoria de subvención del gobierno tripartito de la Xunta de Galicia, regulada en la orden de 14 de febrero del mismo año, en la que se detalla la posibilidad de subvencionar la creación de agencias municipales en materia de empleo, esta subvención estaba destinada a financiar los costes de contratación, locales y equipamientos de estos servicios municipales. Esta primera red se denominó Agencias Municipales de Desarrollo (AMD) y pertenecía a la Red Europea de Intercambios de Información y Experiencias (ERISE). Casi la totalidad de estos primeros técnicos locales se formaron en cursos teórico-prácticos de Agentes de Desarrollo Local (ADL), promovidos por el INEM o la Consellería de Traballo 
e Benestar Social de la Xunta de Galicia, con cofinanciamiento del Fondo Social Europeo.

Más tarde en el año 1991, con el cambio de gobierno en la Xunta de Galicia, esta subvención que financiaba las Agencias de Desarrollo desapareció, y aunque la Diputación de A Coruña y el INEM cofinanciaron algunos años la continuidad de estos servicios, la mayoría se extinguieron. Tan solo se mantuvieron aquellos en los que los ayuntamientos asumieron los costes con sus propios presupuestos municipales. Por lo que, la red de Agencias de Desarrollo Local de Galicia quedó reducida casi en su totalidad.

Será en el año 1998 cuando la Red de Técnicos Locales de Empleo (TLE) subvencionada por la Xunta de Galicia vea la luz.

Tras la publicación de la Orden TAS/360/2008, por la que se modifica la Orden anterior de 1999, en la que se establecen las bases para la concesión de subvenciones, cada servicio Regional de Empleo, al publicar su convocatoria anual, añade funciones específicas en su territorio, baremos diferentes para puntuar los distintos proyectos presentados, teniendo en cuenta criterios como el tamaño el municipio, número de desempleados, existencia o no de otros servicios municipales de promoción económica y empleo, etc., produciéndose diferencias en el porcentaje de costes totales subvencionados, referidos sobre todo a las prórrogas de los contratos. En Galicia, se incorporan además las siguientes funciones:

- Gestionar ayudas para favorecer el empleo.

- Informar a las empresas de los instrumentos de promoción y fomento de la competitividad empresarial disponible.

-Observación estratégica del territorio y realización de estudios con el fin de detectar necesidades formativas y educativas de la población desempleada.

Además, en esta Comunidad Autónoma, se vuelve a una financiación del $80 \%$ y a una duración máxima de cuatro años, como en el resto de las Comunidades Autónomas, porque en la nueva orden ministerial, ya mencionada, desaparece la limitación de cuatro años en los contratos, aunque sigue sin regularse la financiación de los servicios consolidados con personal 
laboral fijo o funcionariado, ni tampoco las actividades ni materiales $\mathrm{o}$ equipamiento de estos servicios.

Ante este panorama evolutivo se puede afirmar que, en los últimos ocho años hasta la actualidad, la contratación del AEDL ha dependido de la subvención que gestionan los Servicios Regionales de Empleo que se publican con una temporalidad anual. La transposición de la Orden TAS/360/2008, de 6 de febrero, regula que estos contratos se puedan renovar todos los años (pero solo en el caso de que exista presupuesto suficiente), lo cual no favorece la creación de puestos de trabajo estables para que el AEDL desarrolle sus funciones con miras de futuro en el ámbito del desarrollo local en los territorios.

A pesar de la evolución y mejora de la normativa (se suprimió la limitación de los cuatro años, se incorporó la dimensión local y adquirieron mayor protagonismo las entidades locales en todo el proceso de concertación territorial y toma de decisiones) los profesionales de AEDL vienen padeciendo una dilatada inestabilidad laboral, situación que se ha podido constatar durante la realización de las entrevistas hechas a los Agentes de los municipios de la provincia de A Coruña.

\subsection{Turismo y Desarrollo Local}

Se aclara que, en el tema de desarrollo local y turismo en España, a día de hoy, no existe un marco teórico consolidado de referencia, aunque se pueden hallar estudios, artículos y obras dedicados a esta materia, en muchos casos, centrados en el desarrollo local del turismo en los espacios rurales.

Álvarez Sousa (2009) argumenta que en España el turismo tuvo unos inicios "anárquicos" ya que no existían unos planes estratégicos que indicasen las pautas a seguir en esta materia. Las prácticas turísticas de los primeros tiempos se desarrollaron obedeciendo, principalmente, a los intereses de los empresarios del sector, faltos de toda ética pragmática que, refugiados tras los objetivos de grandes beneficios económicos, perpetraron agresiones contra el medio ambiente, esquilmado recursos naturales y culturales de difícil recuperación. 
Buen ejemplo de esto se puede observar, como una realidad patente, por toda la costa del litoral mediterráneo, construcciones faraónicas a pie de playa, enclavadas, en no pocas ocasiones, inclusive en parques naturales, menospreciando recursos naturales, culturales e históricos, prevaleciendo los intereses de unos pocos actores sociales frente a los intereses de la población local.

Los cambios producidos en los años ochenta en el sector turístico conllevaron grandes desajustes entre la oferta y la demanda, ello junto al despertar de una conciencia social de protección del medioambiente y la cultura local que además reclamaba un respeto por la calidad de vida de la población local, obligaron a las Administraciones y a los actores sociales, implicados en el sector turístico, a dar una respuesta positiva. Esta nueva realidad emergente impulsó la necesidad de buscar nuevos planes estratégicos consensuados de actuación turística, los cuales habrían de integrar iniciativas empresariales con necesidades básicas de la población local, así como el respeto por el medio ambiente y la cultura local. Así pues, la intervención de la Administración pública en la planificación turística se considerará un proceso necesario para organizar y coordinar los recursos disponibles. Esta intervención pública, dice Ivars, J. (2003) se vio reforzada por las previsiones de crecimiento de la demanda turística, así como las expectativas de iniciativas de desarrollo regional y local, además de existir una clara necesidad de corregir problemas en destinos turísticos ya consolidados con la idea de alcanzar un desarrollo turístico sostenible. Al mismo tiempo, se potencian nuevos modelos de desarrollo "desde abajo", el concepto de desarrollo local se amplía con otras preocupaciones y valores económicos, sociales y medioambientales por lo que se refuerzan los ideales y propuestas para el desarrollo por parte de gobiernos a todas las escalas y se fomentan la cooperación y la "buena gobernanza" (Pike, A. Rodríguez-Pose y Tomaney, 2011).

Se puede afirmar que existe una cercana relación entre el desarrollo de la actividad turística y sus frutos o resultados en el desarrollo local. Algunos estudios realizados en España (Delgado Viña y otros, 2003, Entrena Durán, F., 
2006, Perles Ribes, J.F., 2006, Vargas Sánchez y otros, 2006 y López Guzmán, T.J., 2006) evidencian dicha relación y demuestran que, cuando existen políticas inteligentes de desarrollo local en materia de explotación turística, podemos obtener como resultado productos turísticos viables, sustentables y respetuosos con la comunidad local aprovechando los recursos endógenos, a la par que generadores de beneficios económicos y dinamizadores del territorio. Por otro lado, el turismo nacional e internacional también provoca, en los destinos receptores locales, una expansión de la población local ya que existe una emigración hacia estos lugares por las oportunidades de riqueza que ofrece, crecimiento de los servicios, infraestructuras y empleo.

El resultado de la actividad turística es una convergencia de diversos factores, un acervo de recursos y de interrelaciones entre los diferentes actores sociales implicados, por lo que es necesario acometerla en su conjunto, teniendo en cuenta la unión de elementos. Ivars, A. (2003, p. 39-43) argumenta que los recursos turísticos los podemos encontrar delimitados dentro de un único municipio, pero también puede abarcar un territorio más amplio por lo que interesa confluir diferentes intereses en un mismo proyecto y/o acción turística porque, el espacio turístico y el espacio administrativo, no tienen por qué ser idénticos. Mantero, J.C. (2004) aclara que, en la actividad turística, el proceso de desarrollo se ha de construir sobre la premisa de "pensar global actuar local" y viceversa porque el turismo es una actividad dinámica basada en el flujo de personas. $Y$ advierte de los límites y alcances de la actividad turística en el desarrollo local, ya que su crecimiento no es garantía de desarrollo y ubicar la actividad no es sinónimo de localizar, porque lo importante es la necesidad de "endogenizar apropiadamente en beneficio del territorio" (p. 28).

\subsection{Turismo y Desarrollo Local en Galicia}

El problema, dice Díaz Fernández (2003) es que, en el caso de Galicia, existe una coordinación de la actividad turística internamente fragmentada, 
insuficiencia presupuestaria para cubrir la totalidad de los servicios públicos básicos de ocio turístico, un inadecuado plan de información que no es capaz de reforzar un mensaje favorable hacia el turismo sostenible y una débil estrategia de comunicación en los medios de comunicación.

Se puede observar a continuación, en la tabla 3 , los ayuntamientos de la provincia de A Coruña que han obtenido, desde el 2000 hasta la actualidad, la declaración de "municipio turístico", en concreto solo 13 del total de los 93 que componen la provincia. La ley ha venido a endurecer los requisitos que han de cumplir para conseguir la distinción de municipio turístico.

\section{Tabla 2}

Listado de Ayuntamientos declarados "Municipios Turísticos"

AYUNTAMIENTO

\section{BETANZOS \\ CORCUBIÓN}

FISTERRA

MIÑO

PADRÓN

PONTEDEUME

RIBEIRA

POBRA DO CARAMIÑAL (A)

SANTIAGO DE COMPOSTELA

RIANXO

LAXE

CEDEIRA (SAN ANDRÉS DE TEIXIDO)

MUROS
D.O.G.

$27 / 12 / 2000$

$27 / 12 / 2000$

$28 / 02 / 2000$

$10 / 10 / 2000$

$08 / 02 / 2000$

$08 / 10 / 2001$

$08 / 10 / 2001$

$14 / 08 / 2004$

$24 / 07 / 2003$

$08 / 08 / 2007$

$10 / 12 / 2007$

$09 / 07 / 2009$

$08 / 10 / 2015$

Elaboración propia. Fuente: Xunta de Galicia (Datos extraídos en junio 2016) http://www.turismo.gal/docs/mdaw/mje4/ edisp/turga218674.pdf?langld=es_ES

Ivars (2003) argumenta que la definición de municipio turístico se basa en criterios mayormente cuantitativos, habiendo entre estos municipios grandes variaciones por lo que no deberían ser tratados todos por igual, como unidad, cada uno de ellos tiene sus particularidades. Los municipios turísticos suelen presentar una renta per cápita más alta que aquellos municipios que no lo son, por lo que los primeros reciben más ayudas, además de recibir mayores ingresos en determinadas partidas como el impuesto sobre los bienes inmuebles o por licencias de obra. Por otro lado, nos encontramos con la delimitación del territorio de destino porque el ámbito territorial resultante de 
imagen de destino turístico puede ser distinto de los límites del municipio. "El municipio no delimita el espacio turístico porque éste se configura a partir de los patrones de localización de las actividades turísticas, de las pautas de consumo de los distintos segmentos de demanda y de la imagen del territorio proyectada y percibida" (p. 39), en realidad, lo que nosotros podemos percibir, desde el punto de vista del turista, como un producto global viene determinado por la identificación del espacio o recurso turístico que puede coincidir o no con el espacio de territorio del municipio (división político-administrativa) ya que estos dos espacios no tienen por qué ser idénticos. Es por ello que es esencial poder definir lo que se entiende por municipio turístico a través de unas variables cuantificadoras como son la oferta de alojamiento, ratio de turistas/residentes, tasa de ocupación anual, importancia del turismo en la economía local..., variables que permiten regular los problemas de financiación. Por todo ello, dice Ivars, nos podemos encontrar con regiones turísticas que integren "otras unidades territoriales diferenciadas (...) y que presenten cierto grado de cohesión derivado de la existencia de relaciones funcionales, de la pertenencia a una demarcación político-administrativa o del hecho de compartir una determinada imagen turística" (p.43).

No obstante, y a pesar de las dificultades derivadas de la fragmentación en la gestión administrativa del sector turístico y reducción presupuestaria a nivel municipal, algo que también afecta a su imagen de conjunto como destino, la evolución de crecimiento turístico de Galicia ha ido aumentando en los últimos años. Las cifras revelan dicho incremento y, comparativamente con otras Comunidades Autónomas e incluso con España, su evolución es muy positiva. En concreto Galicia, tal y como ya se reseñó al inicio de este artículo, se muestra como una Comunidad Autónoma con potencialidad turística, en el aumento de la competitividad externa e interna de la propia Comunidad Autónoma, se encuentran economías locales que han de buscar nuevas estrategias e instrumentos de gestión y promoción de las actividades, proyectos y recursos turísticos locales, como pueden ser las colaboraciones. La pujanza del mercado turístico local demanda un papel más relevante del 
consistorio, en el cual el AEDL ha de tener una participación esencial en dicha actividad económica, y tiene mucho que decir al respecto desde su visión estratégica desde abajo, más amplia y cercana a la población local.

\section{Metodología}

La población objeto de estudio son las AEDLs o similar, de los ayuntamientos de la provincia de A Coruña, con competencias en gestión y promoción para el desarrollo socio económico vinculado al turismo en la provincia de A Coruña, siendo éstas las premisas de partida.

La investigación que se aplicó en la primera fase, por el método es exploratoria-descriptiva, y por el tipo de análisis es cualitativa, aplicándose entrevistas bajo el formado de en profundidad semi-estructuradas de aplicación telefónica a los AEDLs, o similar en cada uno de los ayuntamientos.

El período de aplicación de las entrevistas fue de septiembre 2015 a abril 2016. La entrevista se realiza a los 93 municipios para seleccionar la población objeto de estudio (censo definitivo). Con los datos obtenidos de las entrevistas se diseñaron tres mapeos, el primero de ellos para descartar los municipios no válidos para el universo objeto de estudio, y en base a éste se elaboró el segundo y tercer mapeo definitivo para el análisis e interpretación de los datos del censo.

Censo definitivo: 53 casos cumplen las premisas de partida como son: mantener algún tipo de relación desde la AEDL (gestión, organización, promoción y/o colaboración continuada) con las actividades turísticas o disponer de AEDL o similar que cubriese las funciones.

Diseño de las entrevistas bajo el formato de en profundidad semiestructuradas: entrevistas de aplicación telefónica a los 93 ayuntamientos para contactar con el Agente de Desarrollo Local o similar y obtener la información específica. Las preguntas fueron abiertas, siguiendo un guion, con la intención de suscitar amplitud en la respuesta. En muchos casos, las preguntan han llevado a otras no previstas y a obtener más información de la demandada. Se registraron todas las respuestas obtenidas así como los datos facilitados telefónicamente, todo ello nos permitió realizar un total de tres mapeos en 
diferentes fases, el primero de ellos se realiza entre septiembre y diciembre de 2015, el segundo mapeo se cierra en febrero de 2016 y el tercero y definitivo a finales del mes de abril de 2016.

Del censo objeto de estudio, 53 municipios, se comprobaron los datos de contacto obtenidos a través de la web, así como el resto de la información facilitada. También se realiza un rastreo de todo lo publicado en la web y redes sociales relacionado con las actividades y proyectos turísticos locales que confirman gestionar las AEDL.

Tras las entrevistas, en una segunda fase de investigación de tipo cuantitativo, se hace llegar a los AEDLs del censo un cuestionario, del cual se adelantan algunos resultados relevantes, relacionados con el perfil y características de los AEDLs, así como su forma de proceder en la gestión de las actividades turísticas. De los 53 casos que inicialmente conforman el censo quedarán finalmente 49 municipios, debido a que algunos ayuntamientos perdieron su AEDL en el transcurso de la investigación o por considerar que lo que podían aportar en temas de turismo era poco significativo.

\section{Resultados obtenidos}

Presentamos una tabla resumen de algunos de los principales resultados obtenidos a través de los mapeos realizados, en la que se observan las siguientes especificidades diferenciadoras de la situación de partida de las AEDLs:

\section{Tabla 3}

Ayuntamientos de la provincia de A Coruña según situaciones relativas a la $A D L$ y gestión de actividades turísticas

\begin{tabular}{l|l}
\hline $\begin{array}{l}\text { Ayuntamientos que disponen } \\
\text { de AEDL o similar y que } \\
\text { organizan o gestionan }\end{array}$ & $\begin{array}{l}\text { Abegondo, Ames, Aranga, Arzúa, A Baña, } \\
\text { Bergondo, Betanzos, Boqueixón, Cabana de }\end{array}$ \\
actividades turísticas dentro & $\begin{array}{l}\text { Bergantiños, Camariñas, Cambre, Carballo, } \\
\text { Carnota, Carral, Cerceda, Corcubión, }\end{array}$ \\
local políticas de desarrollo & $\begin{array}{l}\text { Culleredo, Dodro, Dumbría, Fene, Ferrol, } \\
\text { lrixoa, Laracha, Lousame, Malpica de }\end{array}$ \\
Representan el 49,46\% & $\begin{array}{l}\text { Bergantiños, Mazaricos, Mesía, Mugardos, } \\
\text { Neda, Negreira, Noia, Oleiros, Pobra do } \\
\text { Caramiñal, Paderne, Porto do Son, Pontes de } \\
\text { García Rodríguez, Rois, Santa Comba, }\end{array}$ \\
\hline
\end{tabular}




\begin{tabular}{|c|c|}
\hline & $\begin{array}{l}\text { Santiso, Sobrado dos Monxes, Toques, } \\
\text { Touro, Val do Dubra, Vilasantar, Vimianzo y } \\
\text { Zas. }\end{array}$ \\
\hline $\begin{array}{l}\text { AEDL que hacen } \\
\text { colaboraciones en materia de } \\
\text { turismo con la Concejalía de } \\
\text { Turismo, Mancomunidad, } \\
\text { Asociaciones o Agrupación } \\
\text { externa al Ayuntamiento }\end{array}$ & $\begin{array}{l}\text { Fisterra, Narón, Padrón, Ponteceso, Ribeira, } \\
\text { Sada y Teo. }\end{array}$ \\
\hline Representan el 7,52\% & \\
\hline $\begin{array}{l}\text { Ayuntamientos que disponen } \\
\text { de AEDL pero no realizan } \\
\text { ninguna actividad relacionada } \\
\text { con el turismo, centrándose } \\
\text { únicamente en la gestión de } \\
\text { empleo, tramitación de } \\
\text { subvenciones o } \\
\text { asesoramiento }\end{array}$ & $\begin{array}{l}\text { Arteixo, Cabanas, Cedeira, Cee, A Coruña, } \\
\text { Laxe, Melide, Miño, Moeche, Monfero, Muros, } \\
\text { Ordes, Oroso, Ortigueira, Oza-Cesuras, O } \\
\text { Pino, Pontedeume, Rianxo, Santiago, } \\
\text { Tordoia, y Vedra. }\end{array}$ \\
\hline Representan el $22,58 \%$ & \\
\hline $\begin{array}{l}\text { En muchos casos suelen ser } \\
\text { Ayuntamientos que tienen } \\
\text { separadas las actividades } \\
\text { turísticas y que suelen ser } \\
\text { realizadas por la Consejería } \\
\text { de Turismo, Cultural o similar. }\end{array}$ & \\
\hline $\begin{array}{l}\text { Ayuntamientos que no } \\
\text { disponen de AEDL ni de } \\
\text { Agente } \\
\text { Representan el } 20,43 \%\end{array}$ & $\begin{array}{l}\text { Ares, Boimorto, Boiro, Brión, A Capela, } \\
\text { Cariño, Cerdido, Coirós, Coristanco, Curtis, } \\
\text { Frades, Mañón, Muxía, Outes, San } \\
\text { Sadurniño, Somozas, Trazo, Valdoviño y } \\
\text { Vilamaior. }\end{array}$ \\
\hline
\end{tabular}

Fuente: elaboración propia, partiendo de los datos extraídos a partir de los mapeos.

Hay que destacar un hecho importante que refleja la situación contractual de algunos ayuntamientos en cuanto a la gestión de las políticas de desarrollo local, ya que se observa que un 20,43\% (19 ayuntamientos) del total de los 93 ayuntamientos de la provincia de A Coruña no disponen del servicio de AEDL. En los casos de los Ayuntamientos de Cariño y Coristanco que si 
disponían anteriormente de AEDL declaran haber perdido este servicio por motivos de restricción presupuestaria, y en el caso del Ayuntamiento de Coirós que no dispone de AEDL propio es porque lo comparte con el Ayuntamiento de Oza-Cesuras, lo mismo ocurre con los Ayuntamientos de Irixoa y Aranga que comparten AEDL, declarando que esta situación les limita alcanzar los objetivos previstos.

De las AEDLs que hacen solo colaboraciones en materia de turismo con la Concejalía de Turismo, Mancomunidad, Asociaciones o Agrupación externa al Ayuntamiento (7,52\%), en algunos casos destacan que antes tenían asumidas muchas más competencias en turismo, como el Ayuntamiento de Sada, el cual gestionaba las rutas de senderismo, la gestión la OIT, la información turística en la web y Facebook, ferias y fiestas gastronómicas y, con la nueva reorganización municipal, después de estas últimas elecciones les suprimieron funciones relacionadas con el turismo en desarrollo local, viéndose limitados a unas colaboraciones puntuales con el proyecto "Mar de Sada" con Acción Costera (GAC) y con el programa europeo de la Reserva de la Biosfera de "Mariñas Coruñesas". En cambio hay otros casos, como la AEDL del Ayuntamiento de Fisterra que se halla en un proceso de reorganización porque quieren relanzar y dar más importancia al turismo desde el desarrollo local.

De los ayuntamientos que disponen de AEDL y realizan funciones relacionadas con el turismo (49,46\%), algunos confirman también haber abandonado, muy a su pesar, proyectos por falta de recursos económicos; destacan los casos del Ayuntamiento de Cerceda y su proyecto de rehabilitación de ferias tradicionales, y el Ayuntamiento de Mugardos con el proyecto Proder "Costa Artabra" y cursos dirigidos al sector hostelero. Resaltar también que aparece como reiterativa la variable "colaboración" ya que algunos proyectos han sido posibles gracias a iniciativas colaborativas con organizaciones externas al Ayuntamiento como son las Mancomunidades, Asociaciones, Agrupaciones, o incluso acudir a la asociación y colaboración entre AEDLs de la misma comarca. 
Al analizar con más detalle la variable "Forma en que se procede en la gestión y promoción de actividades turísticas" por la que también se pregunta en el cuestionario, se observan los siguientes resultados:

\section{Tabla 4}

Frecuencias y porcentajes de la variable Forma en que se procede en la gestión de actividades turísticas

\begin{tabular}{|l|r|r|r|}
\hline & Frecuencia & \multicolumn{1}{|c|}{$\begin{array}{c}\text { Porcentaje } \\
\text { válido }\end{array}$} & $\begin{array}{r}\text { Porcentaje } \\
\text { acumulado }\end{array}$ \\
\hline Válido & $\begin{array}{l}\text { Siempre de forma } \\
\text { independiente }\end{array}$ & 2,1 & 2,1 \\
$\begin{array}{l}\text { Ocasionalmente en } \\
\text { colaboración }\end{array}$ & 29 & 60,4 & 62,5 \\
$\begin{array}{l}\text { Siempre en } \\
\text { colaboración } \\
\text { Total }\end{array}$ & 18 & 37,5 & 100,0 \\
\hline
\end{tabular}

Fuente: elaboración propia

La colaboración preside la forma de trabajar de las AEDLs en cuestiones turísticas, de forma que si sumamos el 37,50\% de "siempre en colaboración" y el $60,42 \%$ de "ocasionalmente en colaboración" da casi el $100 \%$ de los casos, representando un $97,92 \%$. Las cifras muestran lo ya indicado a través de las entrevistas, una forma de abordar o gestionar las actividades turísticas de manera colaborativa, en muchas ocasiones impuesta como estrategia para aunar esfuerzos y sinergias con el intento de alcanzar el éxito en el proyecto o actividad turística.

En el siguiente gráfico de barras horizontales (figura 1) se pueden apreciar los tipos de colaboración a los que han hecho referencia los AEDLs, ante la categoría de opciones que se le planteaban en el cuestionario, pudiendo marcar todas aquellas alternativas de colaboración que considerasen oportunas en cada caso: 


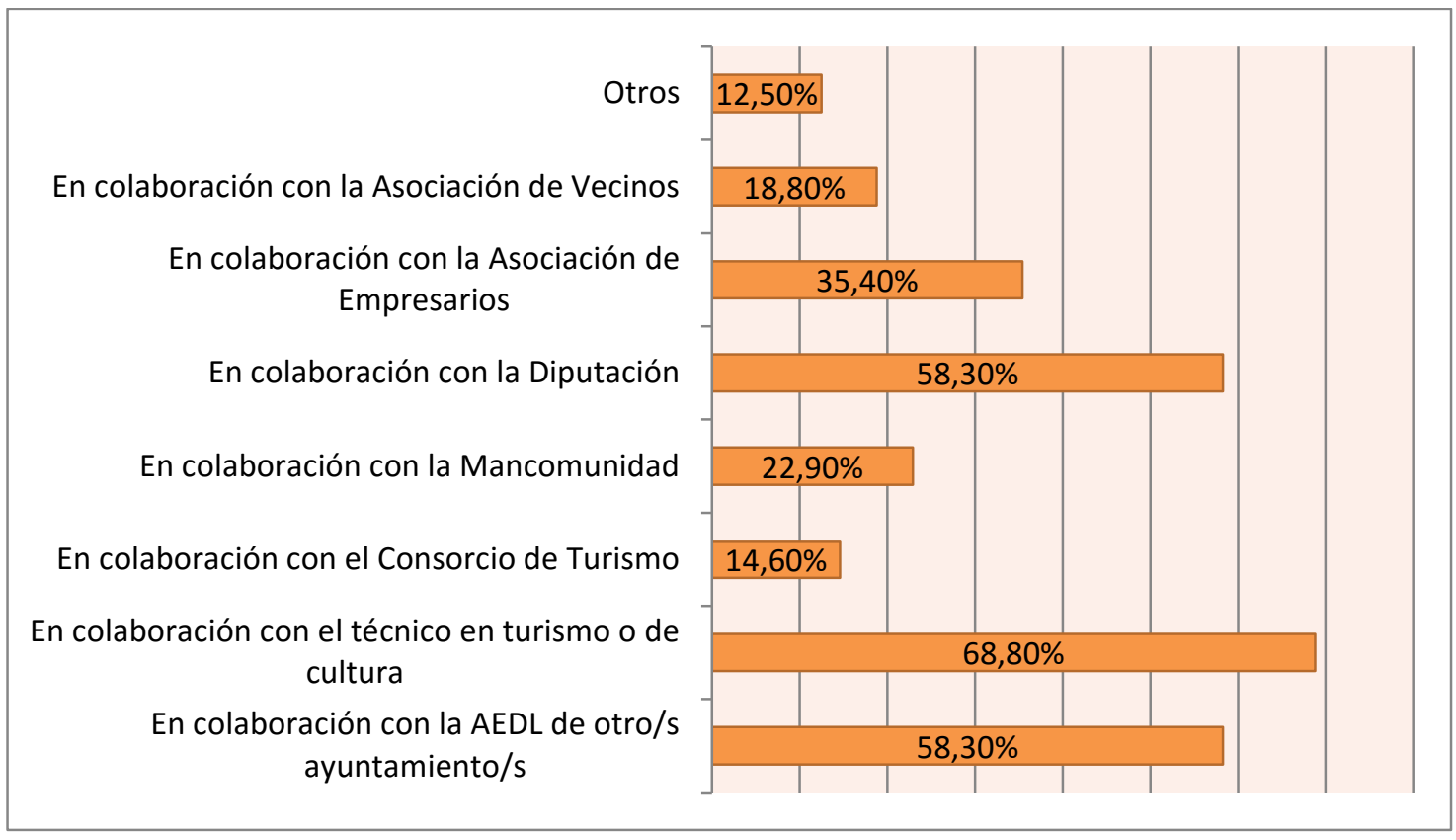

Figura 1. Gráfico de barras horizontales en porcentajes sobre los tipos de colaboraciones.

Fuente: elaboración propia.

Las opciones más valoradas son "en colaboración con el Técnico en Turismo o de Cultura" con un 68,80\%, "en colaboración con la Diputación", "en colaboración con la AEDL de otro/s ayuntamiento/s" coincidiendo en ambos casos con un 58,30\%, y "en colaboración con la Asociación de Empresarios" con un $35,40 \%$.

Otro aspecto importante para comprender esta situación coyuntural mencionada, es observar el número de Agentes por cada ayuntamiento.

\section{Tabla 5}

Frecuencias y porcentajes de la variable Número de Agentes que trabajan en la $A E D L$

\begin{tabular}{|c|c|c|c|c|}
\hline & & Frecuencia & $\begin{array}{c}\text { Porcentaje } \\
\text { válido }\end{array}$ & $\begin{array}{l}\text { Porcentaje } \\
\text { acumulado }\end{array}$ \\
\hline \multirow[t]{4}{*}{ Válido } & 1 & 33 & 68,8 & 68,8 \\
\hline & 2 & 10 & 20,8 & 89,6 \\
\hline & 3 & 5 & 10,4 & 100,0 \\
\hline & Total & 48 & 100,0 & \\
\hline
\end{tabular}

Fuente: elaboración propia 
Al visualizar la tabla 6 de frecuencias, con respecto al número de Agentes que trabajan en la AEDL, un $68,8 \%$ de las mismas disponen de un solo Agente, con dos Agentes un 20,83\% y con tres Agentes un 10,42\%. En los casos que tienen más de un Agente, se pudo comprobar en las entrevistas previas, que tienen separadas las funciones, uno de los Agentes se encarga de las funciones relativas al empleo y el otro de las funciones relacionadas con el desarrollo local, y en los casos de tres Agentes, el tercer Agente está, generalmente, de apoyo para las necesidades de la Agencia o como segundo Agente de Desarrollo Local.

La situación laboral y económica de los AEDLs, se ve reflejada en la dificultad de gestionar todas las funciones y tareas cotidianas de trabajo que han de desarrollar para alcanzar sus objetivos.

Siguiendo con los resultados extraídos de los mapeos, se puede observar que hay una serie de ayuntamientos que antes tenían asumidas la gestión de actividades turísticas y, a día de hoy, les han sido eliminadas.

\section{Tabla 6}

$A E D L$ que antes gestionaban y promocionaban actividades turísticas

\begin{tabular}{l|l}
\hline ADL que antes realizaban & Cariño, Culleredo, Fisterra, Laxe, Miño, \\
actividades relacionadas con el & Oza-Cesuras, Sada, Tordoia \\
sector turístico de forma & \\
continuada, dentro del programa & \\
de desarrollo local, y ahora no & \\
hacen nada o se limitan a & \\
colaboraciones puntuales sin & \\
peso. & \\
Representan un 8,6\% &
\end{tabular}

Fuente: elaboración propia a partir de los mapeos

Estas AEDL que dejaron de realizar funciones relacionadas con el sector turístico de forma continuada, que representan un 8,6\% -8 ayuntamientos- de la totalidad de la provincia de A Coruña, coinciden en afirmar que hay un antes y un después ya que con la crisis han visto reducida sus competencias en desarrollo local, pérdida de personal suficiente para abordar los proyectos y un presupuesto debilitado que les ha obligado a limitar sus funciones a la gestión 
de empleo, tramitación de subvenciones y asesoramiento para iniciativas empresariales, viéndose relegados en funciones. No obstante ellos, que en su día gestionaron actividades turísticas dentro de la promoción económica local del municipio, confirman la importancia de mantener una vinculación con este sector de peso, incluso declarando el firme propósito, como es el caso del Ayuntamiento de Culleredo, de intentar modificar la situación actual porque entienden que es importante potenciar el turismo desde la AEDL. 


\section{Conclusiones}

El papel desempeñado por las AEDL al aplicar las políticas de desarrollo en los municipios, parece tener una desigual práctica en lo tocante al desarrollo de actividades y/o proyectos turísticos locales. Teniendo en cuenta que parece existir diferencias en el desempeño en sus funciones y en las estrategias de desarrollo turístico local, lo cual ha llevado a observar resultados dispares. Se comprueba que el factor tamaño del municipio también está relacionado con el hecho de que el AEDL tenga o no competencias en turismo, observando que los municipios más pequeños (menos de 10.000 habitantes) y situados en zonas de fuerte impacto turístico, el AEDL tiene un papel importante en la gestión y/o promoción de las actividades turísticas.

A la luz de los datos recogidos en las entrevistas y en las respuestas de algunas de las preguntas del cuestionario, se observa que existe una gran cantidad de especificidades particulares, intrínsecas casi a cada Ayuntamiento, como por ejemplo: pendientes de proyectos en vías de diseño, falta de personal (el 68\% de los ayuntamientos de la provincia de A Coruña solo dispone de una persona encargada de las funciones de desarrollo local), escaso presupuesto o recursos económicos limitados, bajas o excedencias sin cubrir, Agentes compartidos entre dos ayuntamientos, acuerdos de colaboración entre municipios, con asociaciones de empresarios 0 Mancomunidades (sinergias frente a proyectos). La causa que alegan principalmente es la situación de crisis económica que vienen sufriendo en los últimos años, lo que ha obligado a muchos ayuntamientos a dejar de gestionar y promocionar actividades relacionadas con el turismo que antes sí hacían, otros se han visto obligados a buscar diversas estrategias para afrontar proyectos y seguir trabajando actividades turísticas. Por lo que se observa la variable "colaboración" como una variable de gran peso, ya que muchos de los proyectos han visto la luz gracias a iniciativas de colaboración con organizaciones externas al ayuntamiento.

La mayoría reconoce la importancia de seguir gestionado las actividades turísticas locales desde al AEDL, ya que permiten dinamizar y 
potenciar el desarrollo económico-social del lugar, resaltando especialmente las actividades y proyectos turísticos relacionados con la sustentabilidad, protección del medio natural o recuperación y restauración de recursos turísticos, ya que proporciona ingresos y empleos al municipio. A pesar de las carencias y dificultades que relacionan, en mayor o menor medida y a veces con ciertas limitaciones, creen cumplir los objetivos socioeconómicos propios de una AEDL.

Consideramos que el AEDL es un profesional que ha de enfocar su trabajo como un ente multidisciplinar y que además está preparado para llevar a cabo, tanto las políticas de empleo como las relacionadas con el desarrollo económico local; pero, sin olvidar que su papel es el de ejercer el impulso y la dinamización del territorio, por lo que aquí entra en juego la dinamización local del turismo. Partiendo del hecho evidente, como ya se vio en la revisión de la literatura, de que el sector turístico es un estandarte en la economía de este país, no se entiende que, en muchos de los casos revisados y descartados para el censo, no se colabore en materia de desarrollo local.

Tras la investigación se ha comprobado la necesidad de cambio de la situación laboral del AEDL, ya que la labor de estos profesionales es primordial para la promoción del desarrollo de las comunidades locales. Las administraciones públicas han de comprender la importancia de consolidar los puestos de AEDL en los ayuntamientos, ya sean éstos grandes o pequeños, y no depender de subvenciones o exiguos presupuestos municipales que no permiten afrontar proyectos y darles continuidad. El éxito de los programas 0 proyectos de desarrollo local dependen, en gran medida, de la capacidad, carga de trabajo y tiempo que se les dedique; tiempo y carga de trabajo que en muchas ocasiones no tienen por falta o escasez de presupuesto y ausencia "obligada" de profesionales para cubrir estas funciones.

Finalmente entendemos que, las diferencias existentes en cuanto a la importancia concedida a la AEDL, dentro de la estructura de la Corporación Municipal, el presupuesto asignado o la posibilidad de sinergias para poner en marcha los proyectos $\mathrm{y} / \mathrm{o}$ actividades, son factores claves para entender la 
ANÁLISIS DEL PERFIL Y SITUACIÓN CONTRACTUAL DE LOS AGENTES DE EMPLEO PÉREZ GARCÍA Antonia

capacidad de gestión y de éxito en el intento de promover el turismo a través del desarrollo local. 


\section{$\underline{\text { 6. Bibliografía }}$}

Alburqueque Llorens, F. (1999) Manual del Agente de Desarrollo Local. Diputación de Barcelona. Colección de Manuales., Centro de Estudios Multidisciplinares. La Paz, Bolivia. Ediciones CEBEM. Primera Edición

Álvarez Sousa, A. (2009) Sociología del turismo. Udima (Universidad a Distancia de Madrid). Edita Centro de Estudios Financieros.

Comisión Europea (2006) Fac Sheet. El enfoque Leader. Guía Básica. Impreso en Bélgica. Documento recuperado 14 de junio de 2016 en http://ec.europa.eu/agriculture/publi/fact/leader/2006_es.pdf

Delgado Viñas y otros (2003) "Turismo y desarrollo local en algunas comarcas de la montaña Cantábrica: Recursos y planificación". Cuadernos de Turismo no 12. Pp. 7-34. Documento recuperado 15 de diciembre de 2015 http://revistas.um.es/turismo/article/view/19321

Díaz Fernández, J.A., Hernández Borge, J. y Patiño Romaris, C.A. (2000) Coord.- Turismo en Galicia. Potencialidades endógenas de desenvolvemento urbano e económico. Edita Excma. Diputación Provincial de Pontevedra. Vigo

Díaz Fernández, J.A. (2003) "La política de desarrollo local y su contribución del desarrollo de las potencialidades del Turismo en Galicia". Revista de desarrollo rural y cooperativismo agrario. № 7. Universidad de Zaragoza. Artículo recuperado 20 de febrero de 2016 http://cederul.unizar.es/revista/num07/03.htm

Entrena Durán, F. (2006) "Turismo rural y desarrollo local: estudio de caso del Sur de España: La Alpujarra". Revista Mexicana de Sociología, 68 (3). UAEM Redalyc.org. Documento recuperado el 15 de diciembre de 2015 http://www.redalyc.org/articulo.oa?id=32112601004

FEPRODEL -Federación Nacional de Profesionales del Desarrollo Local(marzo 2011) "La situación laboral de los y las AEDL's y su realidad profesional" en Afiprodel Recuperado junio de 2016 http://afiprodel.org/afiprodel/bibliografia/

Fuertes, A. M M y Gatica, I. (2008) De la economía global al desarrollo local. Colección desarrollo local, 8. Publicacións de la Universitat de València. González Cardona, Capítulo 11 "El agente de empleo y desarrollo local: competencias profesionales y trabajo en red" (pp. 241-257)

Ivars, J. A. (2003) Planificación turística de los espacios regionales en España. Agencia Valenciana de Turisme. Editorial Síntesis S.A. Madrid 
López Guzman, T.J. y otros (2006) "Las rutas turísticas como motor del desarrollo económico local. La ruta del Tempranillo". Revista de Estudios Turísticos no 167, pp. 131-145. Documento recuperado 15 de diciembre de 2015 https://dialnet.unirioja.es/servlet/articulo?codigo $=2223077$

Mantero, J. C. (2004) "Desarrollo local y actividad turística". Revista, Aportes y Transferencias, vol. 1, pp. 11-38. Centro de Investigaciones Turísticas. Facultad de Ciencias Económicas y Sociales de Universidad de Mar de Plata. (pp.27-28) Recuperado 9 de febrero de 2016. http://nulan.mdp.edu.ar/281/

Perles Ribes, J. F. (2006) "Análisis del impacto económico de eventos: Una aplicación a fiestas populares de proyección turística". Cuadernos de Turismo o 17 pp. 147-166. Documento recuperado 15 de diciembre de 2015. http://papers.ssrn.com/sol3/papers.cfm?abstract id=2213824

Pike A., Rodríguez-Pose A. y Tomaney J. (2011) Desarrollo local y regional. Colección Desarrollo Territorial ํㅡ․ 8ditado por la Universitat de València. Valencia

Redondo Rodríguez de Vera, E. y et. (2000) Manual para la creación y funcionamiento de una agencia de desarrollo local. Unidad de Promoción y Desarrollo adscrita al Área de Fomento de la Excma. Diputación Provincial de Alicante. Consellería d'Ocupació.

Rodríguez Gutiérrez, F. (2001) -eds- Manual de Desarrollo Local. Gijón. Ediciones Trea S.L.

Vargas Sánchez y otros (2007) "Desarrollo del turismo y percepción de la comunidad local: Factores determinantes de su actitud hacia un mayor desarrollo turístico" Empresa Global y mercados locales XXI Congreso Anual AEDEM, Universidad Rey Juan Carlos, Madrid, junio de 2007 https://dialnet.unirioja.es/servlet/articulo?codigo=2517645.

Vázquez Barquero, A. (2000) Desarrollo económico local y descentralización: una aproximación a un marco conceptual. Proyecto CEPAL/GTZ (Comisión Económica para América Latina y el Caribe). Santiago de Chile. Recuperado 9 de febrero de 2016.

http://repositorio.cepal.org/bitstream/handle/11362/31392/S00020088 es.p df?sequence $=1$ 


\section{Webgrafía}

- AFIPRODEL (Asociación Finisterrae de Profesionais de Desenvolvemento Local de Galicia) http://afiprodel.org/afiprodel/afiprodel/listado

- Ley 56/2003 de 16 de diciembre, de Empleo https://www.boe.es/buscar/act.php?id=BOE-A-2003-23102 Recuperado el 7 de mayo de 2016

- Orden Ministerio de Trabajo de 21 de febrero 1986 https://www.boe.es/diario_boe/txt.php?id=BOE-A-1986-5183 Recuperado el 7 de mayo de 2016.

- Orden Ministerio de Trabajo de 12 de abril de 1994 https://www.boe.es/diario_boe/txt.php?id=BOE-A-1994-10061 Recuperado el 7 de mayo de 2016

- Orden Ministerio de Trabajo de 15 de julio de 1999 https://www.boe.es/diario_boe/txt.php?id=BOE-A-1999-16692 Recuperado el 7 de mayo de 2016

- Orden Ministerio de Trabajo de 6 de febrero de 2008 https://www.boe.es/diario_boe/txt.php?id=BOE-A-2008-2777 Recuperado el 7 de mayo de 2016

- Reglamento de Servicios de las Corporaciones Locales. Recuperado el 4 de marzo de 2016 https://boe.es/buscar/pdf/1955/BOE-A-1955-10057-consolidado.pdf 